\title{
Análisis de una Implementación RFID dentro de la Industria Farmacéutica
}

\author{
Analysis of an RFID implementation within the pharmaceutical industry
}

Miguel Ángel Leguizamón Páez ${ }^{1}$, Javier Martínez Pinzón ${ }^{1}$ y Julián Andrés Misnaza Morales ${ }^{1}$

\begin{abstract}
Resumen
¿Cuál o cuáles serían los mecanismos para apoyar a la industria farmacéutica en cuanto a la mejora de sus procesos con herramientas tecnológicas para combatir los medicamentos espurios? Ante este planteamiento se enfrentan las sociedades a nivel mundial, quienes ven con preocupación cómo el mercado de medicamentos falsificados aumenta en una proporción similar a las muertes por su consumo. Este escenario plantea grandes retos para los gobiernos quienes deberán tomar decisiones que intenten detener la fabricación, expendio y consumo de medicamentos espurios, de etiquetado engañoso o de imitación. Existen numerosos riesgos dentro la industria farmacéutica, tales como las imitaciones, procesos incorrectos de fabricación y condiciones inapropiadas de transporte y almacenamiento, que ocasionan riesgos a los diferentes actores como los fabricantes, distribuidores y clientes, de tal manera que pueden llegar a tener pérdidas económicas o afectaciones en la salud. Así, en este documento se pretende mostrar cómo algunas implementaciones que utilizan tecnologías con radiofrecuencia en la industria farmacéutica, ayudan a mitigar la posibilidad de riesgo en las diferentes etapas a lo largo de la cadena de producción y suministro de medicamentos, buscando mejorar la calidad en los procesos y la seguridad en los fármacos.
\end{abstract}

\section{Abstract}

What would be the mechanisms to support the pharmaceutical industry in improving its processes with technological tools to combat spurious drugs? Faced with this approach are the societies worldwide, who see with concern how the market for counterfeit medicines increases in a proportion similar to the deaths by their consumption. This scenario poses great challenges for governments who must make decisions that seek to stop the manufacture, sale and consumption of spurious medicines, misleading or imitation labeling. There are numerous risks within the pharmaceutical industry, such as imitations, incorrect manufacturing processes and inappropriate transport and storage conditions, which pose risks to different actors such as manufacturers, distributors and customers, in such a way that they can lead to losses Economic or health effects. Thus, this document aims to show how some implementations that use radiofrequency technologies in the pharmaceutical industry, help mitigate the possibility of risk at different stages along the production and supply chain of medicines, seeking to improve quality in Processes and drug safety.

\section{Palabras Clave}

Industria farmacéutica; medicamentos espurios; radiofrecuencia; tecnología; trazabilidad.

\section{Key words}

Pharmaceutical Industry; radiofrequency; spurious Medicines; technology; traceability.

${ }^{1}$ Facultad Tecnológica - Ingeniería Telemática, Universidad Distrital Francisco José de Caldas, Bogotá, Colombia.

*Autor correspondiente: cseveriches@gmail.com

Manuscrito recibido 31-03-2017; revisado 30-06-2017; aceptado 07-06-2017.

\section{Introducción}

El presente artículo introduce al lector en la Tecnología de Identificación por Radiofrecuencia - (en adelante RFID) -, para ello se toman como ejemplos algunos casos de éxito en su implementación y uso dentro la industria farmacéutica, en la que se puede evidenciar mejoras en los procesos de distribución [1,2], mitigando diferentes vulnerabilidades por la puesta en marcha de sistemas con tecnología RFID [3].En dicha industria se ponen en funcionamiento diferentes mecanismos de monitoreo que ayudan a hacer un control a los medicamentos en las etapas dentro de la cadena de suminis- 
tros como lo es la logística, el almacenamiento y el transporte, mostrando como la tecnología RFID puede ayudar en esta industria, entre otras.

En la actualidad, existen diversas tecnologías inalámbricas de mayor utilidad para diferentes sectores industriales, que ofrecen alcanzar una mejora y/o actualización de sus procesos; es el caso de las etiquetas RFID cuyas características permiten almacenar información y tener trazabilidad sobre la misma, generando mayores posibilidades en el manejo de la información.

La tecnología RFID se encuentra implementada en diferentes sectores con muestras de efectividad, un ejemplo marcado y con éxito se encuentra en el sector de logística, en el que la tecnología RFID se implementa ayudando a un mejor control de inventarios en el proceso de distribución [4], en el control de acceso al personal por medio de tarjetas con chips o etiquetas, en los aeropuertos o puntos de control donde se implementan pasaportes con esta tecnología.

Dentro de la industria farmacéutica se evidencia según la OMS ${ }^{1}$ [5] una serie de vulnerabilidades relacionadas con la seguridad y la confiabilidad a lo largo de la cadena de suministros de los medicamentos, encontrando productos adulterados o falsificados que generan a la industria pérdidas y a los consumidores riesgos en su salud $[6,7]$. Las autoridades reguladoras y la industria plantean estándares y controles que permiten mejorar la detección de medicamentos SSFFC $^{2}$ y mejorar los procesos de calidad con ayuda de herramientas legales y tecnológicas, dentro de estas últimas se encuentra la tecnología RFID que es el objeto del presente escrito.

\section{Contextualización}

La tecnología es precursora de herramientas, metodologías y paradigmas que llegan a suplir las funciones realizadas por las personas haciendo la vida "simple", es decir, se diseñan útiles, de manera ágil, reduciendo costos operativos, tiempos de ejecución y demás recursos involucrados en los procesos; esto evidentemente sin desligar la responsabilidad del hombre en sus funciones, porque es diseñado por y para el hombre.

Teniendo en cuenta que la tecnología es un beneficio para el hombre, se enfatiza que, al integrar las áreas tecnológicas como la telemática, especialmente la transmisión de datos por medios no guiados dentro de la industria farmacéutica, se logra tener un alto impacto en la vida cotidiana de las personas. Por ejemplo, la sensación de desconfianza en la población con respecto a medicamentos adulterados puede disminuir, generando un aumento en la seguridad del consumo de medicamentos que cuentan con un soporte científico e investigativo. Por otra parte, actores como el gobierno reducen la ilegalidad con un nuevo tipo de control que ayudara a las entidades regulatorias a una mayor precisión de las actividades que se llevan a cabo con respecto a la industria farmacéutica.

\footnotetext{
${ }^{1}$ OMS - Organización Mundial de la Salud.

${ }^{2}$ Medicamentos SSFFC - Medicamentos de calidad sub-estándar, espurios, de etiquetado engañoso, falsificados o de imitación.
}

Las diferentes implementaciones hechas que anteceden a las realizadas con tecnología RFID como el código de barras para el manejo de inventario, junto con la falta de controles que permiten hacer un seguimiento a los productos elaborados durante el proceso hasta llegar al usuario final, abren una puerta a la implementación de nuevas tecnologías que ayudan en la lucha contra los diferentes elementos nocivos que afectan a la industria farmacéutica.

\section{Marco de referencia}

RFID se apoya básicamente en dos componentes: el primero es un chip o etiqueta el cual se adhiere al elemento que se desea monitorear, dicha etiqueta contiene información de identificación y demás sobre el elemento u artículo físico al cual está adherida, desde allí puede emitir y recibir ondas de radiofrecuencia para transmitir cualquier tipo de información, ya sea un número de serie, el valor del artículo, alguna fecha de caducidad e inclusive si su capacidad lo permite la temperatura de este o cambios de la misma en el ambiente [8] (Ver Figura 1). Existen gran variedad de tipos de chips, cada uno diseñado para el entorno en el cual se requiera, estos pueden variar en sus propiedades físicas como la contextura, el tamaño y el material, de esta manera se podrán encontrar de diversas formas y tamaños. Otra de las propiedades de las etiquetas que vale la pena resaltar es la durabilidad, dado que esta puede variar en función de la aplicación o el entorno, es así como las etiquetas permanentes son adecuadas para resistir condiciones ambientales adversas para ellas, algunas pueden ser reutilizables si sus condiciones iniciales lo permiten.

Las etiquetas RFID se clasifican según su comportamiento (activa, pasiva y semi-pasivas). Las etiquetas activas poseen una fuente de energía por tanto son capaces de emitir y recibir señales en un rango definido, cabe destacar que por poseer estas propiedades dentro del mercado son costosos en referencia de los demás. Las etiquetas pasivas sin duda son comunes, su potencia reside en el transmisor del lector, tienen un rango de alcance mucho más corto y su costo es significativamente menor que las activas. Y las etiquetas semi-pasivas se comunican de la misma manera que las etiquetas pasivas, con la diferencia de que poseen una fuente de energía y su rango de transmisión se ubica entre las pasivas y las activas.

El segundo componente es el lector, este es un dispositivo electrónico que emite y recibe señales de radiofrecuencia para leer la información emitida por las etiquetas, dando una gran ventaja en referencia con su antecesor (el código de barras) al poder monitorear diferentes etiquetas al mismo tiempo en un área determinada por el alcance de la frecuencia que estén operando, además de no requerir necesariamente una línea de visión directa con las etiquetas. Estos pueden ser ubicados bajo el piso o en el techo. Asimismo, los sistemas RFID pueden funcionar simultáneamente con redes inalámbricas y suelen integrarse con redes $\mathrm{LAN}^{3}$ inalámbricas para intercambiar datos con los sistemas de los servidores centrales,

\footnotetext{
${ }^{3}$ Redes LAN - Redes de Área Local.
} 
Figura 1. Esquema cómo funciona RFID. Fuente: http://79.170.44.155/hangtags-labels.com/index. php?option=com_content\&view=article\&id=23:how-does-rfid-work\&catid=5:rfid-faos
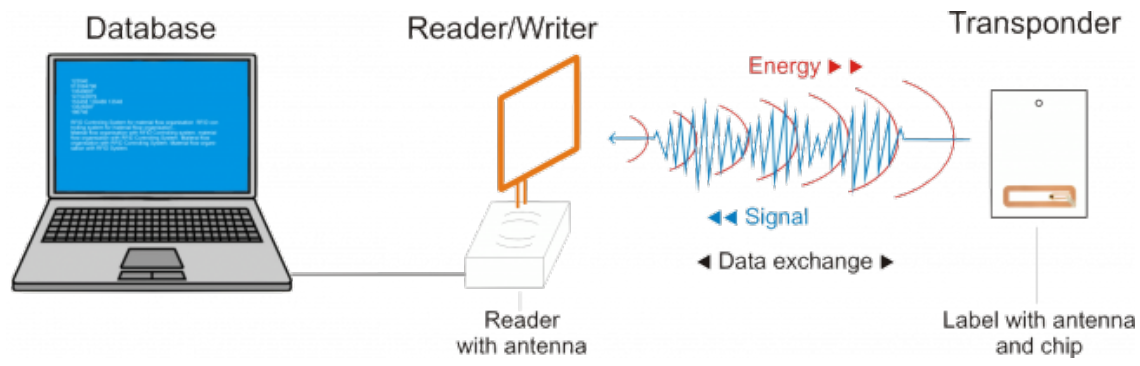

debido a que estas redes no causan interferencias con los sistemas RFID. Los antiguos equipos propietarios de redes inalámbricas de $915 \mathrm{MHz}$ causan interferencias con los sistemas RFID UHF (Ultra High Frecuency - Frecuencia Ultra Alta), aunque actualmente el uso de estos dispositivos es limitado [8]. Estas características demuestran la versatilidad en el uso de esta tecnología en la identificación de artículos, seguir movimientos (trazabilidad), calcular distancias entre puntos por medio de los emisores y receptores, entre otras [9].

Existe un tercer actor que no hace parte íntegra al esquema de componentes para una arquitectura RFID debido a sus variaciones y porque suele estar asociado a implementaciones propias. Este actor es el middleware ${ }^{4}$ cuya función principal es coordinar múltiples lectores que ocupan el mismo espacio físico [10]. En una configuración tradicional el middleware escucha las antenas de un dispositivo lector dentro del rango. Si una etiqueta está presente en el campo de una antena, mientras que el middleware escucha otras antenas, el evento de bajo nivel no puede ser capturado por el middleware. Lo anterior indica que, el middleware entendiéndose como un sistema de software se acopla dentro de su diseño una visión amplia entendiendo a este como una colección de servicios y abstracciones de aplicación específica, de acuerdo con las limitaciones en términos de: a) las capacidades del hardware, y b) los requisitos de aplicación [11].

La manera en cómo evoluciona una tecnología da paso a cómo será el manejo de su seguridad "la tecnología RFID evoluciona rápidamente, las etiquetas se multiplican y se reducen en tamaño, de igual manera las amenazas a las que son susceptibles evolucionan de manera similar" [6]. En cuanto a RFID, el dominio de la información es pública y tiene una gran acogida en diferentes sectores. Son muchas las vulnerabilidades identificadas que tienden a violar la seguridad que brinda esta tecnología, desde ataques triviales como el cambio de etiquetas a productos en almacenes para su robo, hasta creación y desarrollo de software para la intromisión en las frecuencias de transmisión y robo de información que se esté transmitiendo o se encuentre en las etiquetas. Dentro de las vulnerabilidades, se destaca la falta de resistencia a la manipulación como la desactivación o la destrucción de etiquetas, que parte de la capa física, las clonaciones de etiquetas o es-

\footnotetext{
${ }^{4}$ Middleware - Lógica de intercambio de información entre aplicaciones.
}

pionaje de la información, como parte de la capa de transporte, lecturas no autorizadas o modificación de datos en la capa de aplicación; entre otras.

Los sistemas RFID en su mayoría se implementan dentro de diferentes industrias en los procesos logísticos, mejorando el manejo de inventarios, donde existen cambios sustanciales tanto en la reducción de costos de operación como en la precisión y trazabilidad de los productos que manejan las empresas [12]. Un ejemplo de ello se muestra en algunos sectores de la industria colombiana, como es el caso de la Sociedad Portuaria de Cartagena que dentro de su operación incluiye un sistema que permite el monitoreo en tiempo real de activos, mayor atención de los dominios y mejor eficiencia operativa [13]. Este sistema implantado en los camiones de carga fuera del puerto se encarga de vigilar la pila de ingresos al puerto con el fin de agilizar procedimientos portuarios tanto de cargue como descargue.

Otro caso destacado en la industria colombiana es el caso de la empresa $囚$ Familia Sancela quienes integran dentro de sus procesos la tecnología RFID como apoyo a la logística. El objetivo de este sistema en reducir la complejidad con despachos de volúmenes amplios y que requieren gran precisión, además del control y amplía gestión sobre sus inventarios.

De igual manera, en el mundo entero se realiza un trabajo exhaustivo, buscando darle importancia y notoriedad a este tipo de avances tecnológicos. Recientemente en Francia, un grupo biofarmacéutico realiza seguimiento al plasma sanguíneo [2] reemplazando un sistema que se manejaba con código de barras y con el que no se pudo dar solución a problemas como la estandarización en la recolección del plasma y la nula trazabilidad que se tenía. Este nuevo sistema implantado es avalado por las entidades gubernamentales francesas y garantiza un porcentaje de error reducido en la lectura del plasma recibido desde otras instituciones hospitalarias, además permite un nivel de trazabilidad amplio para los investigadores.

\section{Ventajas de la tecnolofía RFID}

El vertiginoso ascenso de RFID es debido a ventajas frente a otras tecnologías que realizan tareas similares, como por ejemplo su predecesor el código de barras, el cual tenía un papel importante apoyando los procesos de identificación. A 
continuación, se evalúan los aspectos que se consideran son superiores en la tecnología RFID [14]:

\subsection{Método de lectura}

Mediante el uso de sensores RFID es útil y de fácil acceso a la información de los productos, debido a que con los lectores tradicionales realizan la recopilación de la información uno a uno, mientras que con RFID se puede leer la información de varios productos ágilmente. Así mismo, la lectura no se restringe cuando la etiqueta se encuentra en malas condiciones, el único requisito es que el chip mantenga su integridad, a diferencia del código de barras donde sus limitantes se remiten al estado en el que se encuentre la etiqueta o el empaque donde está impreso el código y también la visibilidad del mismo [15].

\subsubsection{Velocidad de Lectura}

Debido al uso de sensores, el tiempo de lectura en dispositivos RFID se reduce sustancialmente; en tanto se lee un producto con el lector de código de barras, con RFID se pueden estar procesando estibas enteras de productos que serán rápidamente leídos y pasados al ERP (Enterprise Resource Planning - Planificación de Recursos Empresariales) del cliente.

\subsection{Almacenamiento de datos}

La cantidad de datos que puede almacenar una etiqueta RFID oscila entre los 96bits y 8kbits de información, esto, dependiendo en su mayor medida de las prestaciones del fabricante y el tipo de datos que se deseen almacenar [16]; en comparación con el código de barras que no retiene información y solo se presenta como una secuencia de caracteres que puede ser interpretado por elemento lectores, es decir, se puede entender este código como un sistema en clave morse.

\subsection{Costos}

Al igual que el almacenamiento de información, el costo de cada una de estas soluciones integradas depende de diversos factores. Por lo general, las soluciones son adquiridas como un conjunto, es decir, dentro de esta se incluyen: etiquetas (estas pueden ser de baja, media o alta frecuencia), equipos lectores y el software que administre esta infraestructura. Con esta serie de cambios es seguro que se tenga un impacto en otros sistemas. Por estas razones, es difícil dar un estimado de cuánto pueden llegar a costar este tipo de soluciones. La recomendación es asegurarse de las necesidades requeridas por el negocio para realizar un costeo serio que refleje el objeto de hacer una instalación de este tipo.

\subsection{Seguridad}

La seguridad es un tema sensible en el manejo de la información. Es un hecho que en Colombia existen, y toman fuerza las políticas de protección de datos personales como el Habeas Data, que intenta regular el uso de la información personal por parte de entidades como bancos, compañías de telefonía, etc. Este tipo de leyes promueven el uso responsable de la información de las personas y crea pertenencia sobre la misma; por eso buscan en los sistemas de información un mejor respaldo y una mayor confiabilidad.

Ante esta necesidad inherente, RFID plantea esquemas altamente seguros [17] que eviten la vulneración y alteración de sus etiquetas, y la clonación de la que puedan ser víctimas las mismas.

Uno de los temas controversiales entre los detractores de RFID es la seguridad de la información, para este caso se aborda desde dos enfoques diferentes [18]: el acceso a la información contenida en las etiquetas RFID, que de aquí en adelante se hará referencia como cadena de confianza y el manejo de la información contenida en las etiquetas RFID por parte de las empresas propietarias de las etiquetas. ¿Qué tanto puede afectar el uso de las etiquetas la vida privada de las personas? Lo primero que se debe mencionar es que ningún sistema, hasta este momento, es totalmente seguro ante ataques que puedan llegar a presentarse. Partiendo de esta premisa la pregunta a resolver es: ¿Qué tan seguros son los sistemas RFID?, teniendo en cuenta que RFID es un sistema que tiene sus propias vulnerabilidades [19, 20], generando riesgos en la seguridad. Este es un tema sensible dado el impacto de fiabilidad que tiene sobre los diferentes actores involucrados en la cadena de confianza, no solo sobre el distribuidor sino también sobre el cliente final. Por tal razón, se sigue investigando para mejorar la seguridad en las etiquetas y en todo el sistema $[17,21]$.

Un sistema bastante eficaz para mitigar la seguridad es incluir una firma digital en la etiqueta RFID, con el fin de asegurar la confianza entre el distribuidor y el fabricante, basándose en la técnica de CNT (Call-in the Numeric Token - Ficha numérica por llamada) [22] incluida dentro de múltiples manifiestos realizados para combatir la falsificación de medicamentos y cada empaque se registra en una base de datos del fabricante con un número inequívoco que permite al distribuidor estar seguro de la autenticidad del producto; quedando brechas que no se logran subsanar, tales como: ataque por denegación de servicio, clonación o sobre escritura, entre otras.

Estos inconvenientes son tratados dentro de un sistema TCNT (Time Controlled Numeric Tokens - Fichas numéricas controladas por el tiempo) [23] el cual tiene tres reglas:

- El código de identificación es un número aleatorio único para cada producto en un determinado lote.

- El código de identificación no es un número serial y además debe ser impredecible.

- Debe existir al menos 1000 combinaciones posibles para los productos del lote. Estas combinaciones son almacenadas en la base de datos del proveedor quien crea un método para su posterior consulta de parte del cliente [23].

Los códigos almacenados en base de datos posteriormente son impresos en etiquetas volátiles RFID que son adheridas a los productos que serán distribuidos con la garantía que en caso de ser objetos de alguna manipulación se altera la firma 
y el consumidor sabrá que este producto fue alterado. Como se puede observar tiene los mismos fundamentos de CNT con un alto grado de automatización y mitigación del riesgo.

Otra técnica usada para disminuir la falsificación de etiquetas RFID por clonación se basa en la revisión de eventos generados sobre la etiqueta. Para describir un escenario general de este método se debe decir que todos los registros a los que debe someterse la etiqueta deben ser establecidos con anterioridad y que una verificación anormal indicaría que se intenta producir una falsificación de la etiqueta. Un factor a tener en cuenta en este escenario es la tasa de error de los lectores de las etiquetas o imprecisión identificada, es decir, los casos donde se omite la lectura (conocidos como falsos negativos). Expertos afirman que en el mundo real se observan tasas de lectura a menudo rondan entre el $60-70 \%$ [24]. También se proponen diferentes esquemas de autenticación mutua entre el lector y las etiquetas RFID, para evitar la clonación de las mismas [25].

Como los anteriores ejemplos, existen diferentes protocolos y técnicas de autenticación [21, 25, 26] para mejorar la seguridad de la información entre las etiquetas y los lectores, aunque cada protocolo tiene sus propios inconvenientes y vulnerabilidades como el costo de implementación o el costo computacional. No obstante, se logra evidenciar el dinamismo para generar nuevas soluciones que permitan mitigar los riesgos de seguridad relacionados con un sistema RFID.

En cuanto a la privacidad de la información y el manejo de la misma es un tema que concierne a un ámbito ético por parte de las empresas propietarias de las etiquetas, dado que el usuario final no tiene control sobre estas y se ve vulnerado con respecto a la información recolectada por parte de los vendedores como la localización del usuario u otra información con respecto al producto y que puede ser sensible para el usuario o consumidor.

En un sondeo realizado en la Unión Europea [27] destacan tres preocupaciones principales sobre el uso de RFID:

- Accesos no permitidos a las etiquetas.

- Rastreo de las personas y/o de sus acciones, gustos.

- Uso de los datos para el análisis de comportamientos individuales.

Estas preocupaciones están estrechamente ligadas con que empresas inescrupulosas que pueden atacar un elemento sensible como es la privacidad, por el uso indebido de esta información para beneficio; haciendo campañas de marketing, analizando comportamientos a partir de patrones expresados por las personas, entre otros. No se realizará un análisis profundo sobre los temas éticos dado que no es el objeto del presente artículo, aun así, es importante realizar anotaciones sobre estos aspectos: Algo que deben tener en cuenta los usuarios es que las etiquetas tienen límites determinados como alcance de lectura o una capacidad de almacenamiento. Los gobiernos a nivel mundial toman medidas en el uso indebido de la información de las personas lo que blinda jurídicamente a los usuarios ante ataques a su privacidad.

\section{Industria Farmacéutica}

Dentro de la industria farmacéutica existe una variedad de problemas que se refieren a los procesos relacionados con los medicamentos, la fabricación, la distribución y la venta de estos, que afectan a la industria y a la sociedad [28], esta problemática esta aumentado desde el año 2002 (Ver Figura 2), captando el interés mundial de la industria farmacéutica, que además de las pérdidas económicas, también genera desconfianza por parte de los consumidores y la comunidad en general hacia los laboratorios, las autoridades que regulan la venta y distribución, los gobiernos y demás entes de control. Ejemplo de ello, es una noticia del periódico colombiano "El Tiempo" en su artículo titulado "Hacían medicamentos con cemento, harina, azúcar y ladrillo en Bogotá" publicado en marzo 19 de 2015, este titular evidencia el nivel de riesgo al que se expone en la población Colombiana y de otros países. Es importante el impacto que tiene sobre la población con respecto a la salud pública, ocasionando un significante número de muertes por consumo de este tipo de medicamentos, como se menciona en el portal RFID Pharma Today de mayo del 2012:

"Las naciones unidas estiman que el negocio de la falsificación de drogas ha crecido cerca de $\$ 500$ billones de dólares y es responsable de cerca de 700,000 muertes en un año. En los EE. UU, la desviación y falsificación de drogas le cuesta a la industria aproximadamente 10 por ciento del total de los ingresos".

Con lo anterior, se puede hacer una idea del alcance global que tiene la industria ilegal de medicamentos SSFFC, para lo cual se crean diferentes estrategias para contrarrestar el aumento dado. Uno de los mecanismos que se implementa por parte de la OMS es conformar un grupo de trabajo técnico conjunto con varios estados miembros creando un medio de denuncia y seguimiento de eventos relacionados con medicamentos SSFFC, para mejorar el control con registros actualizados sobre el comportamiento de la industria ilegal en el mundo [28]. Lejos de ser sorprendentes estas cifras, lo que hacen es encender un indicador de alerta hacia las entidades de control de sanidad, que desde hace años combaten esta clase de delitos y que debido a la complejidad del mismo y las estrategias delincuenciales, deben idear maneras efectivas de contrarrestar los fraudes de que afectan la economía y la salud de los usuarios.

Según una estimación publicada en los Estados Unidos de América por el Center for Medicine in the Public Interest, en el año 2016 la cifra de ventas de medicamentos falsificados asciende a 75.000 millones de dólares de los Estados Unidos, lo que equivale a un incremento del $90 \%$ en cinco años [29]. Es importante señalar que los casos de ingesta de medicamentos espurios o falsificados nutren las cifras de fatalidades, otro caso que también vale la pena resaltar es la ingesta de medicamentos en centros hospitalarios inducidos por el personal médico [30] que, por desatenciones suministrar a los 


\section{Tabla 1}

Detecciones de medicamentos falsificados..

\begin{tabular}{|c|c|c|}
\hline Nombre comercial & Informe & $\begin{array}{l}\text { País y año de de- } \\
\text { tección }\end{array}$ \\
\hline $\begin{array}{l}\text { Medicina tradicional } \\
\text { contra la diabetes (us- } \\
\text { ada para bajar el } \\
\text { azúcar en la sangre). }\end{array}$ & $\begin{array}{l}\text { Contenía seis veces } \\
\text { la dosis normal de } \\
\text { glibenclamida (dos } \\
\text { personas murieron, } \\
\text { nueve personas } \\
\text { hospitalizadas) }\end{array}$ & China, 2009 \\
\hline $\begin{array}{l}\text { Metakelfin (contra la } \\
\text { malaria) }\end{array}$ & $\begin{array}{l}\text { Las versiones falsas } \\
\text { descubiertas en } 40 \\
\text { farmacias carecían de } \\
\text { suficiente ingrediente } \\
\text { activo. }\end{array}$ & $\begin{array}{l}\text { República Unida de } \\
\text { Tanzania, } 2009\end{array}$ \\
\hline $\begin{array}{l}\text { Viagra y Cialis (para } \\
\text { la disfunción eréctil) }\end{array}$ & $\begin{array}{l}\text { Las versiones falsas } \\
\text { llegaron de contra- } \\
\text { bando a Tailandia de } \\
\text { fuente desconocida y } \\
\text { país sin identificar. }\end{array}$ & Tailandia, 2008 \\
\hline $\begin{array}{l}\text { Xenical (contra la } \\
\text { obesidad) }\end{array}$ & $\begin{array}{l}\text { Versiones falsificadas } \\
\text { vendidas en EE.UU. a } \\
\text { través de sitios web } \\
\text { que operan en otros } \\
\text { lugares, pero que no } \\
\text { contienen el ingredi- } \\
\text { ente activo. }\end{array}$ & Estados Unidos, 2007 \\
\hline
\end{tabular}

Figura 2. Comparativo por años sobre incidentes con respecto a medicamentos falsificados Fuente: http://WwW . psi-inc.org/spanish/incidentTrends.cfm

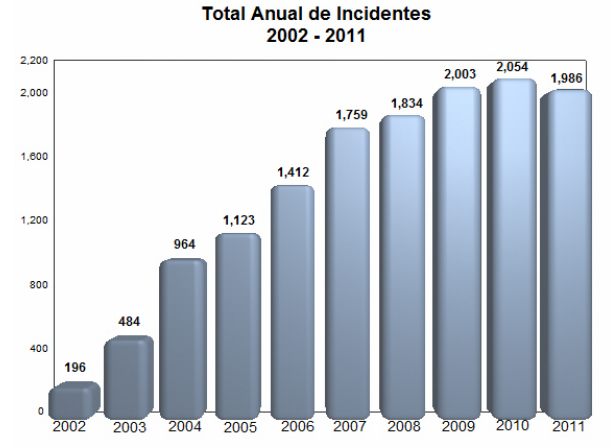

pacientes medicamentos equivocados o en mal estado. $\mathrm{Mu}-$ chos de estos casos son documentados a nivel mundial, como por ejemplo el registrado en España por El Diario en una publicación hecha el 4 de mayo de 2016 "Un informe publicado esta semana indica que más de un $9,5 \%$ de las muertes en Estados Unidos se deben a mala praxis, solo detrás de las enfermedades coronarias y el cáncer, mala praxis en la que se incluye el suministro de medicamentos erróneos". Además, resalta que "estos errores son la tercera causa de muerte más común en Estados Unidos. Cada año mueren por este motivo más de 250.000 personas". Ante estos señalamientos los investigadores encargados de recoger dichas cifras en Estados Unidos y a nivel mundial indican que, es claramente necesario un método que limite el margen de error producto de los errores humanos [31]. Teniendo en cuenta que estos son inevitables, algunos centros hospitalarios realizan esfuerzos para incluir dentro sus organizaciones estrategias que incluyan tecnologías de radiofrecuencia como RFID, buscando que el personal encargado de suministrar los medicamentos a los pacientes reduzca el riesgo de equivocaciones y mediante el uso de un sensor escanee los mismos para saber ¿qué es? y ¿en qué condiciones se encuentran? los medicamentos para ser suministrados, inclusive información de periodicidad en el suministro y ¿en qué momento se debe volver a suministrar?

De igual forma, el diario colombiano El Espectador en su columna "Medicamentos, 15 mil errores en nueve años" publicada el 11 de abril de 2016, resalta hechos similares que tienen lugar en la praxis hospitalaria colombiana y señala que: en el lapso comprendido entre enero de 2008 a septiembre de 2013, se cometió un poco más de 9.000 errores en medicación. Estos errores son adjudicados al personal de enfermería y a los médicos quienes recetan la medicación; también se pudo establecer que los casos más comunes para estos errores se 
encontraban en el suministro de medicamentos para ojos y oídos y que, aunque en su mayoría no son casos mortales se debe contar con elementos que contribuyan al mejoramiento de la prestación del servicio y reduzcan estos indicadores.

\subsection{Fabricación}

La fabricación de medicamentos se basa en la normatividad de cada país y en las recomendaciones hechas por las instituciones reglamentarias como la OMH (Organización Mundial de la Salud, en español OMS) o la PAHO (Organización Panamericana de la Salud). Estas organizaciones indican que es responsabilidad de laboratorios autorizados por entidades gubernamentales, quienes avalan una serie de condiciones como salubridad, lineamientos médicos y científicos para producir medicamentos que cumplan con altos estándares de calidad. Por tanto, se debe contar con un personal altamente calificado además de instalaciones y equipos que soporten este nivel de exigencia. Cuando se habla de medicamentos espurios todo este conducto regular es omitido y no se presenta ninguno de estos escenarios, en algunos casos el problema es tan profundo que se omiten los límites éticos trazados por una sociedad, en otros casos leves, pero igualmente serios, las falsificaciones pueden contener el principio activo incorrecto o el principio activo correcto en cantidades incorrectas, sin dañar de manera significativa al consumidor.

Las incautaciones de medicamentos que incumplen con los estándares de fabricación y no avalados por entidad gubernamental son más del $50 \%$ de medicamentos del mercado en el 2015 (Ver Figura 3). Esta cifra muestra el riesgo al que se expone la sociedad por parte de organizaciones estructuradas que trafican con medicamentos que incumplen los estándares. Asimismo, es importante resaltar que con respecto a los años anteriores se tiene un aumento significativo en el número de decomisos de medicamentos falsificados, aspecto preocupante, que indica que deben intensificarse los esfuerzos para frenar el consumo de este tipo de medicamentos.

\section{RFID dentro de la industria farmacéutica}

La inclusión de la tecnología RFID en la industria farmacéutica es un gran apoyo dentro de las diferentes etapas de la cadena de suministros, donde se pueden implementar varias soluciones que ayudan a mitigar los riesgos [32]. Entre los aportes significativos se encuentran:

- La reducción de fármacos falsificados y adulterados en el mercado.

- La sensación de seguridad entre los compradores y consumidores de medicamentos quienes estarán seguros que el producto que se está ingiriendo es el correcto.

- El monitoreo de enfermedades de alto riesgo con mayor agilidad por parte del personal médico.
Figura 3. Incautaciones de falsificaciones realizadas en el año 2015. Decomisos de más de 1000 unidades de dosificación es clasificado como un incidente de tipo comercial, de igual manera incidentes de menos de 1000 unidades están catalogados como "no comercial". http: / / www.psi-inc.org/ spanish/incidentTrends.cfm

\section{Incautaciónes de Falsificaciónes 2015}

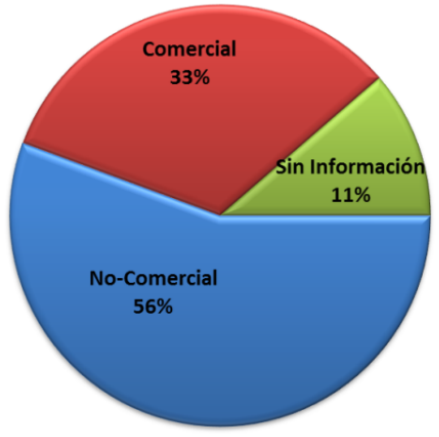

- La reducción de errores por parte del personal médico en el suministro de medicamentos a pacientes en las clínicas y hospitales, entre otros beneficios.

- Debido al aumento en la falsificación de medicamentos varios países enfocan su atención en esta problemática y ponen a disposición la infraestructura tecnológica y las leyes necesarias para combatir este flagelo. De hecho, Estados Unidos cuentan con un ambicioso proyecto llamado Electronic Pedigree (e-Pedrigree - eP-) [33], correspondiente a una legislación vigente en varios estados y la definición de un estándar avalado por la GS1 5. Electronic Pedigree básicamente es un documento electrónico en formato XML que provee un historial acerca de la "vida" de un medicamento y declara que el origen del mismo es conocido, por tanto, se encuentra apto para su circulación, comercialización y consumo por parte de los clientes [34]. En este registro se encuentran las fechas y los nombres de quienes manipulan el medicamento. Otra iniciativa importante es la que lidera la Unión Europea [33, 35] PoD (Pointof-Dispense Authentication).

- Autenticación Punto de Entrega) en cabeza de su organismo EFPIA (European Federation of Pharmaceutical Industries and Associations - Federación Europea de Industrias y Asociaciones Farmacéuticas), encargada

\footnotetext{
${ }^{5} \mathrm{GS} 1$ - Organización privada dedicada a la elaboración y aplicación de servicios mundiales y soluciones para mejorar la eficiencia y visibilidad de las cadenas de abastecimiento, la oferta y la demanda a nivel mundial y en todos los sectores
} 
de verificar la autenticidad de los medicamentos desde el punto inicial o fabricante hasta el punto final o distribuidor mediante el uso de números únicos de serie registrados en códigos QR ${ }^{6}$. Sin embargo, GS1 define una arquitectura [36] que permite gradualmente la integración con RFID, marcando una tendencia hacia esta tecnología. Los factores importantes al momento de integrar RFID son los costos operativos que involucra el pasarse a una tecnología que modifica los mecanismos tradicionales y el otro factor importante es la colaboración de los diferentes actores, la acción colectiva de los involucrados para manejar la autenticidad y seguridad de la información es vital para que el proceso sea eficiente y efectivo [36].

En la actualidad existen diferentes procesos dentro de la industria farmacéutica integrando soluciones telemáticas para la tecnología RFID [37], es el caso del proceso de producción donde por medio de la serialización en masa e identificación automática (Auto-ID) el etiquetado y embalaje de productos se logran de manera ágil, reduciendo tiempos y mejorando el control de los productos. La fabricación de medicamentos está constituida por una serie de pasos complejos. Iniciando en procesos investigativos en los laboratorios de una compañía de la industria farmacéutica [38]. Luego se realiza un proceso de selección de compuestos que pueden contrarrestar los efectos nocivos de la enfermedad en cuestión. Los laboratorios cuentan con enormes bases de datos de proteínas y compuestos. Esta elección es realizada por un robot que selecciona los componentes necesarios para crear el nuevo medicamento. Luego, se realizan procesos de pruebas y optimización bajo ambientes controlados dentro del laboratorio. Finalmente, se procede a la producción y empaquetamiento.

Con el desarrollo de tecnologías para la serialización en masa e identificación automática (Auto-ID), en particular de identificación por radiofrecuencia (RFID), procesos como el etiquetado y embalaje son ágiles, reduciendo tiempos en los procesos. Por ello, la industria farmacéutica motiva al uso de tecnologías RFID. Entre las principales razones se encuentran:

- RFID tiene un gran potencial para afrontar la lucha contra la falsificación $[39,40]$ porque permite autenticar bajo diversos métodos seguros los productos [41]; como la firma digital, el estampado cronológico, entre otros.

- El potencial que se encuentra en RFID radica en su fácil acoplamiento con otras tecnologías como NFC (Near Field Communication - Comunicación en campo cercano). La tecnología NFC permitirá integrar funcionalidades de RFID en un teléfono celular y funcionar como lector [42].

- RFID brinda altas posibilidades de trazabilidad, generando que los fabricantes de fármacos tengan control sobre

\footnotetext{
${ }^{6}$ Código QR - Del inglés Quick Response Code - Código de Respuesta Rápida
}

sus medicamentos y oportunidades para médicos y pacientes de tener un método de control y seguimiento a las medicinas en un tratamiento [40].

- La inclusión de etiquetas RFID disminuye el suministro erróneo de medicamentos a pacientes hospitalarios disminuyendo las cifras fatales por estos casos.

\subsection{Distribución de medicamentos}

La distribución de medicamentos es la cadena de suministro, donde se encuentran subprocesos como el almacenamiento y el transporte luego de fabricados a su destino: una farmacia, un hospital o directamente al consumidor. Debido a que los medicamentos requieren de condiciones especiales de tratamiento, la logística es fundamental y debe garantizar que los productos a almacenar y transportar no sean alterados por ningún factor.

Dentro de la industria de la logística existe una rama que importante y delicada relacionada con la cadena de frío [1]. En la logística y almacenamiento de medicamentos existen diferentes factores como la temperatura, la fecha de vencimiento, la humedad y, la luz, entre otros [43]que influyen en el deterioro de los productos, un ejemplo son medicamentos como vacunas, medicina externa, medicina oral o la sangre. En la actualidad, existen sistemas que permiten realizar un seguimiento de la temperatura, por termostatos, infrarrojos o cámaras que miden la temperatura, teniendo en cuenta lo anterior, las etiquetas RFID mejoran los controles en tiempo real, por ser sensibles a los cambios de temperatura [44], monitoreando desde una plataforma central la información recolectada, con ayuda de una red de sensores inalámbricos y sistema de sensores RFID. Lo anterior, ayudar a reducir el riesgo de ocurrencias, controlando en periodos de tiempos cortos los diferentes factores ambientales que degradan los medicamentos en las diferentes etapas del proceso [39].

Las etiquetas RFID se integran con la tecnología ZigBee [43] basadas en el estándar 802.15.4 de la IEEE. Estas etiquetas manejan un bajo costo por sus características, como una baja complejidad a diferencias de otro tipo de redes inalámbricas de área personal - WPAN - (como Bluetooth y WiFi), bajo poder de transmisión; por medio de los diferentes sensores y las etiquetas RFID realizan la comunicación de los datos dentro del sistema, manteniendo actualizado en tiempo real la información relevante para el control de los medicamentos. Esta forma de concebir la interacción entre los objetos, es importante por los avances de las plataformas Web para mantener conexión con diversos objetos llamado "internet de las cosas - IoT-".

Por lo anterior, es útil para la industria farmacéutica usar los componentes RFID porque las ventajas son amplias desde la cadena de suministro de los medicamentos al realizar monitoreo constante sobre embalajes y empaquetado de los productos hasta el uso combinado con ZigBee y la instalación de sensores en las etiquetas RFID para realizar un monitoreo constante sobre la salud de pacientes que necesiten un tratamiento farmacológico vigilado. Adicionalmente, estos elementos 
cuentan con sistemas fiables, seguros que cubren rangos mayores de distancia y geolocalizables.

La integración de la tecnología RFID en cada uno de los procesos mencionados anteriormente en la industria farmacéutica conlleva a nuevos retos en el momento de la implementación como lo es un cambio en la infraestructura, inversión en nuevos equipos lectores, middleware, etiquetas y demás artefactos necesarios para una correcta implementación. No obstante, se debe tener en cuenta el final de las etiquetas, la reutilización de las mismas, el reciclaje que se les puede llegar a dar enmarcado dentro de un modelo de sostenibilidad [33].

\section{Conclusiones}

RFID es un gran aporte tecnológico que impactará al sector de la salud por su versatilidad. El éxito de RFID radica fundamentalmente en la relación costo beneficio frente a su referente más cercano el código de barras, tanto en sus propiedades de seguridad y trazabilidad.

Colombia debe generar estrategias a nivel legislativo y tecnológico, que impulse a la industria farmacéutica para la implementación de nuevas estrategias que mitiguen los riesgos en la cadena de suministros de fármacos y disminuya la ilegalidad ocasionada por la falsificación de medicamentos. Por ello, el uso de tecnologías RFID es relevante y es un elemento principal que brinda soporte a nuevos mecanismos de la seguridad.

Se puede establecer que importantes actores a nivel mundial ven a la tecnología RFID como una gran apuesta a corto plazo; prueba de ellos es la inclusión de estándares en la norma ISO 18000 y su vinculación con proyectos estatales como (e-Pedrigree - eP-) en los Estados Unidos, y en actos legislativos de la Unión Europea para la regulación de los dispositivos para proteger la seguridad de las personas y de los productos.

Como sistema, RFID, realiza grandes esfuerzos en el mejoramiento de estándares de calidad y seguridad, haciendo especial énfasis en el trato de datos personales, incluyendo en sus sistemas complejos esquemas de seguridad que disminuyen las vulnerabilidades y reducen la posibilidad de ataques.

El uso de la tecnología RFID en el ámbito farmacéutico y en particular con lo vinculado a los temas hospitalarios tendría un alto impacto en la reducción de incidentes relacionados con el suministro de medicamentos erróneos, puesto que disminuiría el margen de error humano, brindándole al personal médico herramientas de alta tecnología para el cuidado de los pacientes.

Las posibilidades que ofrece RFID son amplias e incluyen el monitoreo de enfermedades que se deben inspección por parte del personal médico, lo que implica un mayor monitoreo y una reducción sustancial de visitas hospitalarias.

\section{Agradecimientos}

Los autores agradecen a sus familias por el gran apoyo con su tiempo y paciencia para la consecución de este artículo; además agradecen a la Universidad Distrital Francisco José de Caldas Facultad Tecnológica quien los acogió en su amable seno para el crecimiento personal y profesional de los autores.

\section{References}

[1] F. Li and Z. Chen, "Brief analysis of application of rfid in pharmaceutical cold-chain temperature monitoring system," in Proceedings 2011 International Conference on Transportation, Mechanical, and Electrical Engineering (TMEE), pp. 2418-2420, Dec 2011.

[2] B. Violino, "Grupo biofarmacéutico francés hace el seguimiento del plasma sanguíneo." RFID Journal Español, Agosto 2015.

[3] M. Laniel and I. Uysal, "Rfid system optimization for item-level pharmaceutical serialization," in 2013 IEEE International Conference on RFID-Technologies and Applications (RFID-TA), pp. 1-6, Sept 2013.

[4] T. M. Choi, "Coordination and risk analysis of vmi supply chains with rfid technology," IEEE Transactions on Industrial Informatics, vol. 7, pp. 497-504, Aug 2011.

[5] A. M. de la Salud, "Productos médicos de calidad subestándar, espurios, de etiquetado engañoso, falsificados o de imitación: Informe de la directora general," 2014.

[6] E. Tardif, "Medicamentos falsificados: una píldora difícil de tragar y un reto sanitario global," Anuario Español de Derecho Internacional, vol. 27, pp. 591-613, 2011.

[7] O. M. de la Salud, "La falsificación de medicamentos: una amenaza creciente," tech. rep., Organización Mundial de la Salud, Abril 2010.

[8] Intermec, "Conceptos básicos de rfid: Conocimiento y uso de la identificación por radiofrecuencia," tech. rep., Intermec, 2007.

[9] S. M. Roy and N. C. Karmakar, "Introduction to rfid systems," Handb. Smart Antennas RFID Syst, pp. 11-51, July 2011.

[10] APCglobal, "Epc information services (epcis) version 1.0.1 specification," tech. rep., APCglobal Inc., Sept. 2007.

[11] J. S. Ting, "Arquitectura de software para los actuales sistemas ciber-físicos," Ingenierías USBmed, vol. 2, no. 1, pp. 29-32, 2011.

[12] Y. Miaji, M. A. A. Mohamed, and N. B. Daud, "Rfid based improving supply chain traceability," in 2013 IEEE International Conference on RFID-Technologies and Applications (RFID-TA), pp. 1-6, Sept 2013.

[13] R. Journal, "La terminal de contenedores de colombia pone en marcha la tecnología de rfid,” Ene. 2012.

[14] R. Derakhshan, M. E. Orlowska, and X. Li, "Rfid data management: Challenges and opportunities," in 2007 
IEEE International Conference on RFID, pp. 175-182, March 2007.

[15] C. Poetker, D. Rodgers, M. Sarachman, K. Traub, S. Ackley, and D. Anarkat, "Gs1 rfid/barcode interoperability guideline,” tech. rep., GS1 Global Office, 2016.

[16] R. Journal, "Preguntas frecuentes."

[17] C. H. Cho, K. H. Do, J. W. Kim, and M. S. Jun, "Design of rfid mutual authentication protocol using time stamp," in 2009 Fourth International Conference on Computer Sciences and Convergence Information Technology, pp. 1047-1051, Nov 2009.

[18] INTECO, "Guía sobre seguridad y privacidad de la tecnología rfid," tech. rep., Instituto Nacional de Tecnologías de la Comunicación (INTECO), 2010.

[19] M. Lehtonen, F. Michahelles, and E. Fleisch, "How to detect cloned tags in a reliable way from incomplete rfid traces," in 2009 IEEE International Conference on RFID, pp. 257-264, April 2009.

[20] L. Mirowski and J. Hartnett, "Deckard: A system to detect change of rfid tag ownership," International Journal of Computer Science and Network Security, vol. 7, pp. 89-98, July 2007.

[21] Y. Liu, "An efficient rfid authentication protocol for lowcost tags," in 2008 IEEE/IFIP International Conference on Embedded and Ubiquitous Computing, vol. 2, pp. 180185, Dec 2008.

[22] R. G. Johnston, "An anticounterfeiting strategy using numeric tokens," International Journal of Pharmaceutical Medicine, vol. 19, pp. 163-171, Jun 2005.

[23] E. Nilsson, B. Nilsson, and E. Järpe, "A pharmaceutical anti-counterfeiting method using time controlled numeric tokens," in 2011 IEEE International Conference on RFIDTechnologies and Applications, pp. 343-347, Sept 2011.

[24] S. R. Jeffery, M. Garofalakis, and M. J. Franklin, “Adaptive cleaning for rfid data streams," in Proceedings of the $32 N d$ International Conference on Very Large Data Bases, VLDB '06, pp. 163-174, VLDB Endowment, 2006.

[25] Y. C. Huang and J. R. Jiang, "Efficient ultralightweight rfid mutual authentication," in 2014 IEEE International Conference on Internet of Things (iThings), and IEEE Green Computing and Communications (GreenCom) and IEEE Cyber, Physical and Social Computing (CPSCom), pp. 102-108, Sept 2014.

[26] A. B. Jeng, L.-C. Chang, and T.-E. Wei, "Survey and remedy of the technologies used for rfid tags against counterfeiting," in 2009 International Conference on Machine Learning and Cybernetics, vol. 5, pp. 2975-2981, July 2009.

[27] R. Acierno, M. Maffia, L. Mainetti, L. Patrono, and E. Urso, "Rfid-based tracing systems for drugs: Technological aspects and potential exposure risks," in 2011
IEEE Topical Conference on Biomedical Wireless Technologies, Networks, and Sensing Systems, pp. 87-90, Jan 2011.

[28] J. Harris, P. Stevens, and J. Morris, "Gato por liebre. la amenaza de las medicinas falsas," tech. rep., Health Issues Internacional Policy Network, 2009.

[29] P. Shetty, "Medicinas falsas: hechos y cifras," Marzo 2011.

[30] G. Q. Huang, Z. Qin, T. Qu, and Q. Dai, "Rfid-enabled pharmaceutical regulatory traceability system," in 2010 IEEE International Conference on RFID-Technology and Applications, pp. 211-216, June 2010.

[31] R. A. G. Gao, C. DesRoches, and A. Jha, "The role of information systems in healthcare: Current research and road ahead," Information Systems Research, vol. 22, pp. 419-428, 2011.

[32] M. Potdar, E. Chang, and V. Potdar, "Applications of rfid in pharmaceutical industry," in 2006 IEEE International Conference on Industrial Technology, pp. 2860-2865, Dec 2006.

[33] M. L. Pardal, M. Harrison, S. Sarma, and J. A. Marques, "Expressive rfid data access policies for the pharmaceuticals supply chain," in 2013 IEEE International Conference on RFID (RFID), pp. 199-206, April 2013.

[34] T. Inaba, "Inference of product quality by using rfidenabled traceability information a study on the us pharmaceutical supply chain," in 2009 IEEE International Conference on RFID, pp. 298-305, April 2009.

[35] J. Dalli, "Ensuring patients have access to safe medicines," tech. rep., European Stakeholder Model, 2011.

[36] R. T. Wigand, D. M. Mande, and J. D. Wood, "Information management and tracking of drugs in supply chains within the pharmaceutical industry," in 2011 Eighth International Conference on Information Technology: New Generations, pp. 500-507, April 2011.

[37] T. Engel, S. Lunow, J. Fischer, F. Koebler, S. Goswami, and H. Krcmar, "Value creation in pharmaceutical supply chains using customer- centric rfid applications," in Smart SysTech 2012; European Conference on Smart Objects, Systems and Technologies, pp. 1-8, June 2012.

[38] S. Campillo, "Del laboratorio a tu botiquín: ¿cómo se fabrican los medicamentos?." Hipertextual, Diciembre 2014.

[39] C. C. Drugs, "A report of the food and drug administration," tech. rep., U. S. Department of Health and Human Services, 2004.

[40] B. King and X. Zhang, "Securing the pharmaceutical supply chain using rfid," in 2007 International Conference on Multimedia and Ubiquitous Engineering (MUE'07), pp. 23-28, April 2007. 
[41] M. Lehtonen, T. Staake, and F. Michahelles, From Identification to Authentication - A Review of RFID Product Authentication Techniques, ch. 9, pp. 169-187. Berlin, Heidelberg: Springer Berlin Heidelberg, 2008.

[42] N. Forum, "Nfc and contactless technologies," 2007.

[43] Z. Jianbo and Y. Qun, "Design of pharmaceutical drugs security based on rfid sensor networks," in 2014 9th International Conference on Computer Science Education, pp. 1010-1013, Aug 2014.

[44] R. Bhattacharyya, C. Floerkemeier, S. Sarma, and D. Deavours, "Rfid tag antenna based temperature sensing in the frequency domain," in 2011 IEEE International Conference on RFID, pp. 70-77, April 2011. 